\title{
ROUGH SUBALGEBRAS OF SOME BINARY ALGEBRAS CONNECTED WITH LOGICS
}

\author{
WIESŁAW A. DUDEK AND YOUNG BAE JUN
}

Received 5 May 2004 and in revised form 29 October 2004

Properties of rough subalgebras and ideals of some binary algebras playing a central role in the theory of algebras connected with different types of nonclassical logics are described.

\section{Introduction}

In 1982, Pawlak introduced the concept of a rough set (see [10]). This concept is fundamental for the examination of granularity in knowledge. It is a concept which has many applications in data analysis (see [11]). Rough set theory is applied to semigroups and groups (see $[7,8])$. In this paper, we apply the rough set theory to $\mathrm{BCH}$-algebras, that is, to algebras of type $(2,0)$ satisfying some simple axioms. Since these algebras play a central role in the theory of many types of algebras connected with different types of nonclassical logics obtained results can be extended to the theory of such algebras. In particular, to the theory of BCK/BCI/BCC-algebras [9], QBC-algebras [4, 5], Hilbert algebras [1] and prelogics [2].

\section{Preliminaries}

Many types of algebras connected with nonclassical logics (cf., e.g., [1, 6, 9]) have one binary operation $*$ and one constant denoted by 0 or by 1 . This operation with the corresponding constant is connected by the axiom $x * x=0$ (or by $x * x=1$ ) and satisfies the identity $(x * y) * z=(x * z) * y$ (resp., $x *(y * z)=y *(x * z))$. Moreover, in such algebras one can define a natural partial order for which the constant 0 is the smallest (or minimal) element (resp., 1 is the largest (or maximal) element). In many cases these partial orders are dual. Also some algebras are dual. This means that a very important role in the study of such algebras is played by algebras with the constant 0 satisfying the identity $x * x=0$ and may by other simple axioms. One of such algebras is a $\mathrm{BCH}$-algebra.

Recall that a $\mathrm{BCH}$-algebra is an algebra $(X, *, 0)$ of type $(2,0)$ satisfying the following axioms: for every $x, y, z \in X$,

(H1) $x * x=0$,

(H2) $(x * y) * z=(x * z) * y$, 


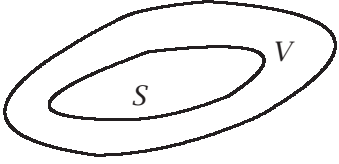

(a)

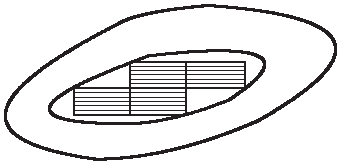

(b)

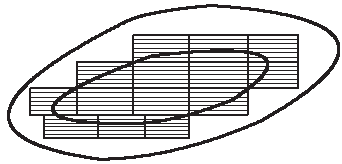

(c)

Figure 2.1 (a) $S \subset V$. (b) $E^{-}(S) \subseteq S$. (c) $S \subseteq E^{+}(S)$.

(H3) $x * y=0$ and $y * x=0$ imply $x=y$.

In a BCH-algebra $X$, the following holds: for all $x, y \in X$,

(u1) $x * 0=x$,

(u2) $0 *(x * y)=(0 * x) *(0 * y)$.

For any $\mathrm{BCH}$-algebra $X$, the relation $\leq$ is defined by $x \leq y$ if and only if $x * y=0$ is a partial order on $X$. A nonempty subset $S$ of a BCH-algebra $X$ is said to be a subalgebra of $X$ if $x * y \in S$ whenever $x, y \in S$. A nonempty subset $A$ of a $\mathrm{BCH}$-algebra $X$ is called an ideal of $X$ if it satisfies

(I1) $0 \in A$,

(I2) $x * y \in A$ and $y \in A$ imply $x \in A$ for all $x, y \in X$.

Note that an ideal of a BCH-algebra $X$ may not be a subalgebra of $X$. An ideal $A$ of a $\mathrm{BCH}$-algebra $X$ is said to be closed if $0 * x \in A$ for all $x \in A$. Note that every closed ideal of a $\mathrm{BCH}$-algebra is a subalgebra, but the converse may not be true. An ideal $A$ of a $\mathrm{BCH}$ algebra $X$ is called a translation ideal of $X$ if whenever $x * y \in A$ and $y * x \in A$, then $(x * z) *(y * z) \in A$ and $(z * x) *(z * y) \in A$ for all $x, y, z \in X$. Note that $\{0\}$ and $X$ itself are translation ideals of $X$.

Let $V$ be a set and $E$ an equivalence relation on $V$ and let $\mathscr{P}(V)$ denote the power set of $V$. For all $x \in V$, let $[x]_{E}$ denote the equivalence class of $x$ with respect to $E$. Define the functions $E^{-}, E^{+}: \mathscr{P}(V) \rightarrow \mathscr{P}(V)$ as follows (see Figure 2.1): for all $S \in \mathscr{P}(V)$,

$$
E^{-}(S)=\left\{x \in V \mid[x]_{E} \subseteq S\right\}, \quad E^{+}(S)=\left\{x \in V \mid[x]_{E} \cap S \neq \varnothing\right\}
$$

The pair $(V, E)$ is called an approximation space. Let $S$ be a subset of $V$. Then $S$ is said to be definable if $E^{-}(S)=E^{+}(S)$ and rough otherwise. $E^{-}(S)$ is called the lower approximation of $S$ while $E^{+}(S)$ is called the upper approximation. Obviously, if $x$ is in $E^{+}(S)$, then $[x]_{E}$ is contained in $E^{+}(S)$. Similarly, if $x$ is in $E^{-}(S)$, then $[x]_{E}$ is contained in $E^{-}(S)$.

\section{Rough subalgebras and ideals}

Let $E$ be a congruence relation on a $\mathrm{BCH}$-algebra $X$, that is, $E$ is an equivalence relation on $X$ such that $(x, y) \in E$ implies $(x * z, y * z) \in E$ and $(z * x, z * y) \in E$ for all $z \in X$. We denote by $X / E$ the set of all equivalence classes of $X$ with respect to $E$, that is, $X / E:=\left\{[x]_{E} \mid x \in X\right\}$. Throughout this section $X$ is a BCH-algebra, and $E$ is a congruence relation on $X$. 
A special role is played by relations determined by ideals, that is, relations $\Theta$ of the form

$$
(x, y) \in \Theta \Longleftrightarrow x * y \in U, y * x \in U,
$$

where $U$ is an ideal. If $U$ is a translation ideal of $X$, then $\Theta$ is a congruence on $X$ (see [12]). In this case we say also that $\Theta$ is a congruence relation related to $U$.

Theorem 3.1. $[0]_{E}$ is a closed ideal of $X$.

Proof. If $x \in[0]_{E}$, then $(x, 0) \in E$, so $(0,0 * x)=(x * x, 0 * x) \in E$. Hence $0 * x \in[0]_{E}$. Let $x, y \in X$ be such that $x * y \in[0]_{E}$ and $y \in[0]_{E}$. Then $(x * y, 0) \in E$ and $(y, 0) \in E$. Since $E$ is a congruence relation on $X$, it follows from (u1) that $(x * y, x)=(x * y, x *$ $0) \in E$ so that $(x, 0) \in E$, that is, $x \in[0]_{E}$. Therefore $[0]_{E}$ is a closed ideal of $X$.

Define an operation $*$ on $X / E$ by $[x]_{E} *[y]_{E}=[x * y]_{E}$ for all $[x]_{E},[y]_{E} \in X / E$. Observe that $\left(X / E, *,[0]_{E}\right)$ may not be a $\mathrm{BCH}$-algebra, because $X / E$ does not satisfy the condition (H3).

Theorem 3.2. The following are equivalent:

(1) for all $x, y \in X, x * y \in[0]_{E}$, and $y * x \in[0]_{E}$ imply $(x, y) \in E$,

(2) $E$ is regular, that is, $[x]_{E} *[y]_{E}=[0]_{E}=[y]_{E} *[x]_{E}$ implies $[x]_{E}=[y]_{E}$,

(3) $\left(X / E, *,[0]_{E}\right)$ is a $\mathrm{BCH}$-algebra.

Proof. $(1) \Rightarrow(2)$. Suppose $[x]_{E} *[y]_{E}=[0]_{E}=[y]_{E} *[x]_{E}$. Then $[x * y]_{E}=[0]_{E}=[y *$ $x]_{E}$, and so $(x * y, 0) \in E$ and $(y * x, 0) \in E$. It follows from $(1)$ that $(x, y) \in E$, that is, $[x]_{E}=[y]_{E}$.

(2) $\Rightarrow(3)$. Obvious.

$(3) \Rightarrow(1)$. Let $x, y \in X$ be such that $x * y \in[0]_{E}$ and $y * x \in[0]_{E}$. Then $[x]_{E} *[y]_{E}=$ $[x * y]_{E}=[0]_{E}=[y * x]_{E}=[y]_{E} *[x]_{E}$. It follows from $(\mathrm{H} 3)$ that $[x]_{E}=[y]_{E}$, that is, $(x, y) \in E$. This completes the proof.

For any nonempty subsets $A$ and $B$ of $X$, we define

$$
A * B:=\{x * y \mid x \in A, y \in B\} .
$$

Proposition 3.3. Let $A$ and $B$ be nonempty subsets of $X$. Then the following hold:

(1) $E^{-}(A) \subseteq A \subseteq E^{+}(A)$,

(2) $E^{+}(A \cup B)=E^{+}(A) \cup E^{+}(B)$,

(3) $E^{-}(A \cap B)=E^{-}(A) \cap E^{-}(B)$,

(4) $A \subseteq B$ implies $E^{-}(A) \subseteq E^{-}(B)$ and $E^{+}(A) \subseteq E^{+}(B)$,

(5) $E^{+}\left(E^{+}(A)\right)=E^{+}(A)$ and $E^{-}\left(E^{-}(A)\right)=E^{-}(A)$,

(6) $E^{-}(A \cup B) \supseteq E^{-}(A) \cup E^{-}(B)$,

(7) $E^{+}(A \cap B) \subseteq E^{+}(A) \cap E^{+}(B)$,

(8) $E^{+}(A) * E^{+}(B)=E^{+}(A * B)$,

(9) $E^{-}(A) * E^{-}(B) \subseteq E^{-}(A * B)$ whenever

$$
E^{-}(A) * E^{-}(B) \neq \varnothing, \quad E^{-}(A * B) \neq \varnothing .
$$


Table 3.1

\begin{tabular}{llllll}
\hline$*$ & 0 & 1 & 2 & 3 & 4 \\
\hline 0 & 0 & 0 & 0 & 0 & 4 \\
1 & 1 & 0 & 0 & 1 & 4 \\
2 & 2 & 2 & 0 & 0 & 4 \\
3 & 3 & 3 & 3 & 0 & 4 \\
4 & 4 & 4 & 4 & 4 & 0 \\
\hline
\end{tabular}

The proof of Proposition 3.3 is straightforward.

Definition 3.4. A nonempty subset $S$ of $X$ is called an upper (resp., a lower) rough subalgebra (or, (closed) ideal) of $X$ if the upper (resp., nonempty lower) approximation of $S$ is a subalgebra (or, (closed) ideal) of $X$. If $S$ is both an upper and a lower rough subalgebra (or, (closed) ideal) of $X$, it is said that $S$ is a rough subalgebra (or (closed) ideal) of $X$.

Theorem 3.5. Every subalgebra $S$ of $X$ is a lower rough subalgebra if only $E^{-}(S) \neq \varnothing$.

Proof. Let $S$ be a subalgebra of $X$ and let $E^{-}(S) \neq \varnothing$. Taking $A=B=S$ in Proposition $3.3(9)$, we have

$$
E^{-}(S) * E^{-}(S) \subseteq E^{-}(S * S) \subseteq E^{-}(S)
$$

because $S$ is a subalgebra of $X$. Hence $E^{-}(S)$ is a subalgebra of $X$, that is, $S$ is a lower rough subalgebra of $X$.

The following example shows that the lower approximation of a subalgebra may be the empty set.

Example 3.6. Let $X=\{0,1,2,3,4\}$ be a BCH-algebra with the Cayley Table 3.1 (see [12]). Then $U=\{0,1,2\}$ is a translation ideal of $X$ (see [12, Example 3.3]). Let $\Theta$ be a congruence relation on $X$ related to $U$. Then all equivalence classes of $X$ are $[0]_{\Theta}=[1]_{\Theta}=$ $[2]_{\Theta}=\{0,1,2\},[3]_{\Theta}=\{3\}$, and $[4]_{\Theta}=\{4\}$. It is not difficult to see that $S=\{0,2\}$ is an example of a subalgebra of $X$ for which $\Theta^{-}(S)=\varnothing$.

THEOREM 3.7. Every subalgebra of $X$ is an upper rough subalgebra of $X$.

Proof. Let $S$ be a subalgebra of $X$. We show that $E^{+}(S)$ is a subalgebra of $X$. Let $x, y \in$ $E^{+}(S)$. Then $[x]_{E} \cap S \neq \varnothing$ and $[y]_{E} \cap S \neq \varnothing$. Thus there exist $a_{x}, a_{y} \in S$ such that $a_{x} \in$ $[x]_{E}$ and $a_{y} \in[y]_{E}$. It follows that $\left(a_{x}, x\right) \in E$ and $\left(a_{y}, y\right) \in E$ so that $\left(a_{x} * a_{y}, x * y\right) \in E$, that is, $a_{x} * a_{y} \in[x * y]_{E}$. On the other hand, $S$ is a subalgebra of $X$, so $a_{x} * a_{y} \in S$. Hence $a_{x} * a_{y} \in[x * y]_{E} \cap S$, that is, $[x * y]_{E} \cap S \neq \varnothing$. This shows that $x * y \in E^{+}(S)$. Therefore $S$ is an upper rough subalgebra of $X$.

The following example shows that the converse of Theorem 3.7 may not be true. Hence we know that the notion of an upper rough subalgebra is an extended notion of a subalgebra. 
Example 3.8. Let $X$ and $U$ be as in the previous example. Consider a subset $S=\{2,3\}$ of $X$ which is not a subalgebra of $X$. Then

$$
\Theta^{+}(S)=\left\{x \in X \mid[x]_{\Theta} \cap S \neq \varnothing\right\}=\{0,1,2,3\},
$$

which is a subalgebra of $X$.

Lemma 3.9 [3, Proposition 3]. Let $A$ be a subalgebra of $X$. Then $A$ is a closed ideal of $X$ if and only if $y * x \in X \backslash A$ whenever $x \in A$ and $y \in X \backslash A$.

THeORem 3.10. If $A$ is a closed ideal of $X$, then the nonempty lower approximation of $A$ is a closed ideal of $X$, that is, $A$ is a lower rough closed ideal of $X$.

Proof. Let $A$ be a closed ideal of $X$. Then $A$ is a subalgebra of $X$, and so $E^{-}(A)$ is a subalgebra of $X$ (see Theorem 3.5). Let $x, y \in X$ be such that $x \in E^{-}(A)$ and $y \in X \backslash E^{-}(A)$. If $y * x \notin X \backslash E^{-}(A)$, that is, $y * x \in E^{-}(A)$, then

$$
[y]_{E} *[x]_{E}=[y * x]_{E} \subseteq A .
$$

Let $a_{y} \in[y]_{E}$. Then for every $a_{x} \in[x]_{E}$, we have $a_{y} * a_{x} \in[y]_{E} *[x]_{E} \subseteq A$. Since $a_{x} \in$ $[x]_{E} \subseteq A$ and $A$ is an ideal of $X$, it follows from (I2) that $a_{y} \in A$ so that $[y]_{E} \subseteq A$. Therefore $y \in E^{-}(A)$, which is a contradiction. Thus, by Lemma 3.9, we know that $E^{-}(A)$ is a closed ideal of $X$.

Proposition 3.11. If $\Theta$ is the congruence relation on $X$ related to $\{0\}$, then every subset of $X$ is definable.

Proof. Let $A$ be a subset of $X$. Note that

$$
\begin{aligned}
{[x]_{\Theta} } & =\{y \in X \mid(x, y) \in \Theta\} \\
& =\{y \in X \mid x * y=0, y * x=0\}=\{y \in X \mid x=y\}=\{x\}
\end{aligned}
$$

for all $x \in X$. Hence

$$
\begin{aligned}
& \Theta^{-}(A)=\left\{x \in X \mid[x]_{\Theta} \subseteq A\right\}=A, \\
& \Theta^{+}(A)=\left\{x \in X \mid[x]_{\Theta} \cap A \neq \varnothing\right\}=A .
\end{aligned}
$$

This completes the proof.

Proposition 3.12. If $\Theta$ is the congruence relation on $X$ related to $X$, then $X$ is definable.

Proof. It is straightforward, since

$$
[x]_{\Theta}=\{y \in X \mid(x, y) \in \Theta\}=\{y \in X \mid x * y \in X, y * x \in X\}=X
$$

for all $x \in X$.

Theorem 3.13. Let $\Theta$ be the congruence relation on $X$ related to a translation ideal $U$ of $X$. If $A$ is a closed ideal of $X$ containing $U$, then $\Theta^{+}(A)$ is a closed ideal of $X$. 
Proof. Obviously $0 \in \Theta^{+}(A)$. If $x \in \Theta^{+}(A)$, then $[x]_{\Theta} \cap A \neq \varnothing$. Hence there exists $a \in A$ such that $a \in[x]_{\Theta}$. Since $A$ is a closed ideal of $X$, we have $0 * a \in A$, and so

$$
0 * a \in\left([0]_{\Theta} *[x]_{\Theta}\right) \cap A=[0 * x]_{\Theta} \cap A .
$$

Thus $[0 * x]_{\Theta} \cap A \neq \varnothing$, which means $0 * x \in \Theta^{+}(A)$. Let $x, y \in X$ be such that $x * y \in$ $\Theta^{+}(A)$ and $y \in \Theta^{+}(A)$. Then $[y]_{\Theta} \cap A \neq \varnothing$ and $[x * y]_{\Theta} \cap A \neq \varnothing$. It follows that there exist $a, b \in A$ such that $a \in[x * y]_{\Theta}$ and $b \in[y]_{\Theta}$ so that $(a, x * y) \in \Theta$ and $(b, y) \in \Theta$. Then $(b, y) \in \Theta \Rightarrow y * b \in U \subseteq A$, and $(a, x * y) \in \Theta$ implies $(x * y) * a \in U \subseteq A$. Since $a, b \in A$ and $A$ is a closed ideal of $X$, it follows from (I2) that $y \in A$ and $x * y \in A$ so that $x \in A$. Note that $x \in[x]_{\Theta}$, thus $x \in[x]_{\Theta} \cap A$, that is, $[x]_{\Theta} \cap A \neq \varnothing$. Therefore $x \in \Theta^{+}(A)$. This completes the proof.

A map $f: X \rightarrow Y$ of BCH-algebras is called a homomorphism if

$$
f(x * y)=f(x) * f(y) \quad \forall x, y \in X .
$$

Note that the kernel of $f$, denoted by $\operatorname{Ker}(f)$, is a translation ideal of $X$ (see [12, Theorem 3.6]).

Proposition 3.14. If $f: X \rightarrow Y$ is a homomorphism of $\mathrm{BCH}$-algebras, then

$$
f(A * \operatorname{Ker}(f))=f(A) \subseteq f\left(E^{+}(A)\right)
$$

for all subsets $A$ of $X$.

Proof. Let $y \in f(A)$. Then $y=f(a)$ for some $a \in A$. Since $A \subseteq E^{+}(A)$ by Proposition 3.3(1), it follows that $y=f(a) \in f\left(E^{+}(A)\right)$ so that $f(A) \subseteq f\left(E^{+}(A)\right)$. Since $0 \in \operatorname{Ker}(f)$, it follows from (u1) that

$$
y=f(a)=f(a * 0) \in f(A * \operatorname{Ker}(f))
$$

so that $f(A) \subseteq f(A * \operatorname{Ker}(f))$. Now, if $z \in f(A * \operatorname{Ker}(f))$, then $f(x)=z$ for some $x \in$ $A * \operatorname{Ker}(f)$. Hence $x=a * b$ with $a \in A$ and $b \in \operatorname{Ker}(f)$. Then

$$
z=f(x)=f(a * b)=f(a) * f(b)=f(a) * 0=f(a) \in f(A),
$$

and so $f(A * \operatorname{Ker}(f)) \subseteq f(A)$. This completes the proof.

Proposition 3.15. Let $f: X \rightarrow Y$ be a homomorphism of $\mathrm{BCH}$-algebras. If $\Theta$ is the congruence relation on $X$ related to $\operatorname{Ker}(f)$, then $f(A)=f\left(\Theta^{+}(A)\right)$ for all subsets $A$ of $X$.

Proof. Using Proposition 3.14, we have $f(A) \subseteq f\left(\Theta^{+}(A)\right)$. Let $y \in f\left(\Theta^{+}(A)\right)$. Then $y=$ $f(x)$ for some $x \in \Theta^{+}(A)$. Hence $[x]_{\Theta} \cap A \neq \varnothing$, and so there exists $a \in A$ such that $a \in[x]_{\Theta}$. Now $a \in[x]_{\Theta}$ implies $(a, x) \in \Theta$, that is, $a * x \in \operatorname{Ker}(f)$ and $x * a \in \operatorname{Ker}(f)$. Thus $f(a) * f(x)=f(a * x)=0$ and $f(x) * f(a)=f(x * a)=0$. It follows from (H3) that $f(x)=f(a)$ so that $y=f(x)=f(a) \in f(A)$. Therefore $f\left(\Theta^{+}(A)\right) \subseteq f(A)$. This completes the proof. 
Remark 3.16. Results presented in this paper are formulated for $\mathrm{BCH}$-algebras, but the axioms (H1), (H2), (H3) defining BCH-algebras and identities (u1), (u2) satisfied by these algebras are not used in all proofs. This means that part of our results is valid for algebras satisfying only two from three axioms defining $\mathrm{BCH}$-algebras.

\section{Acknowledgment}

The second author was supported by Korea Research Foundation Grant KRF-2001-005D00002.

\section{References}

[1] W. J. Blok and D. Pigozzi, Algebraizable logics, Mem. Amer. Math. Soc. 77 (1989), no. 396, vi+78.

[2] I. Chajda and R. Halaš, Algebraic properties of pre-logics, Math. Slovaca 52 (2002), no. 2, 157175.

[3] M. A. Chaudhry and H. Fakhar-Ud-Din, Ideals and filters in BCH-algebras, Math. Japon. 44 (1996), no. 1, 101-111.

[4] R. Halaš and J. Ort, QBCC-algebras inherited from qosets, Math. Slovaca 53 (2003), no. 4, 331340.

[5] Standard QBCC-algebras, Demonstratio Math. 36 (2003), no. 1, 1-10.

[6] K. Iséki and S. Tanaka, An introduction to the theory of BCK-algebras, Math. Japon. 23 (1978/1979), no. 1, 1-26.

[7] N. Kuroki, Rough ideals in semigroups, Inform. Sci. 100 (1997), no. 1-4, 139-163.

[8] N. Kuroki and J. N. Mordeson, Structure of rough sets and rough groups, J. Fuzzy Math. 5 (1997), no. $1,183-191$.

[9] J. Meng and Y. B. Jun, BCK-Algebras, Kyung Moon Sa, Seoul, 1994.

[10] Z. Pawlak, Rough sets, Internat. J. Comput. Inform. Sci. 11 (1982), no. 5, 341-356.

[11] _ Rough Sets-Theoretical Aspects of Reasoning about Data, Theory and Decision Library. Series D: Systems Theory, Knowledge Engineering and Problem Solving, vol. 9, Kluwer Academic, Massachusetts, 1991.

[12] E. H. Roh, S. Y. Kim, and Y. B. Jun, On a problem in BCH-algebras, Math. Japon. 52 (2000), no. 2, 279-283.

Wiesław A. Dudek: Institute of Mathematics, Wrocław University of Technology, Wybrzeże Wyspiańskiego 27, 50-370 Wrocław, Poland

E-mail address:dudek@ulam.im.pwr.wroc.pl

Young Bae Jun: Department of Mathematics Education, Gyeongsang National University, Jinju 660-701, Korea

E-mail address: ybjun@gsnu.ac.kr 


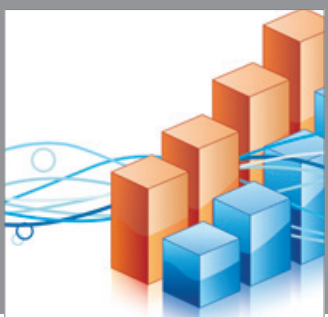

Advances in

Operations Research

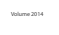

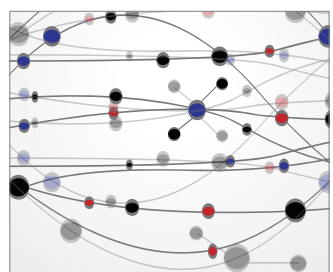

\section{The Scientific} World Journal
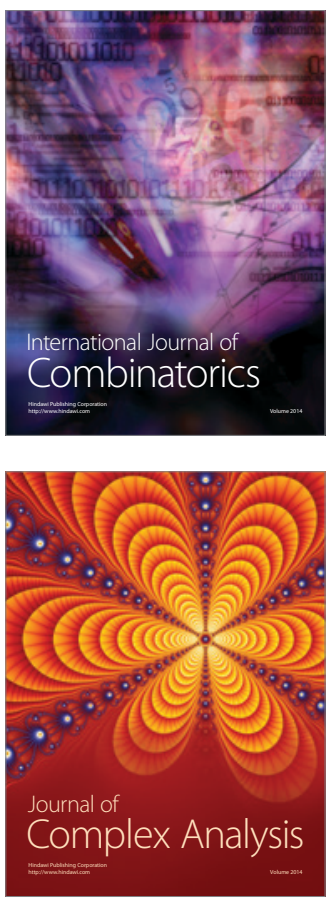

International Journal of

Mathematics and

Mathematical

Sciences
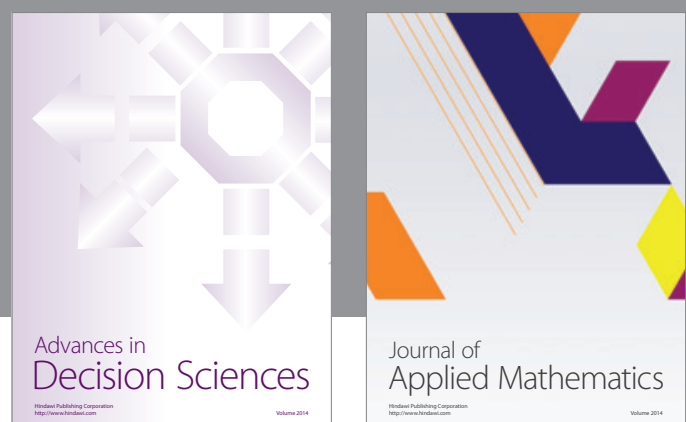

Journal of

Applied Mathematics
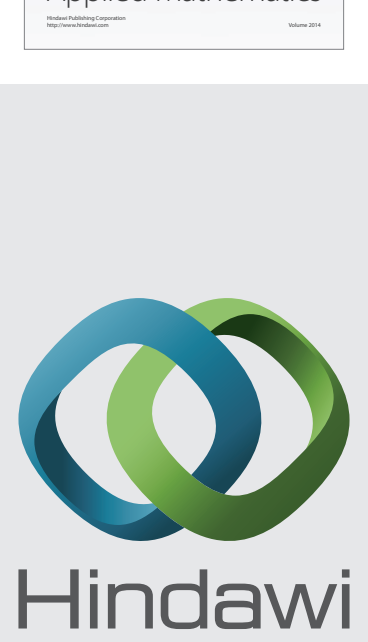

Submit your manuscripts at http://www.hindawi.com
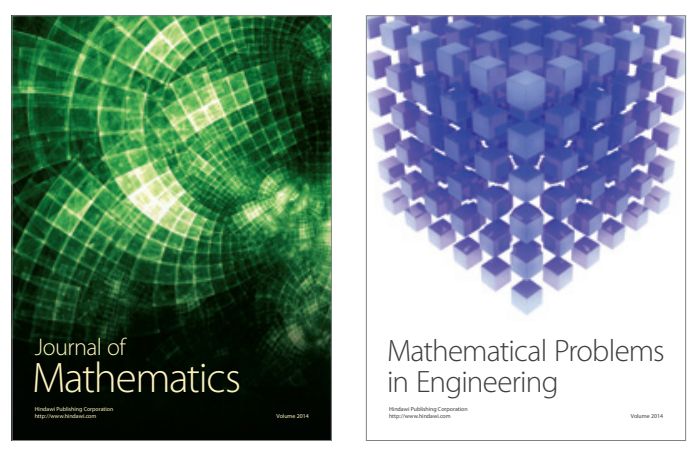

Mathematical Problems in Engineering
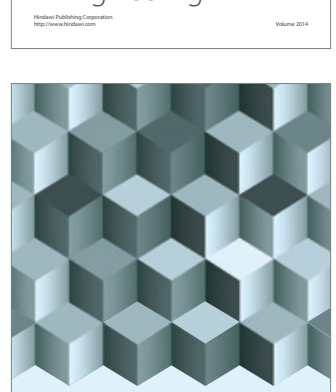

Journal of

Function Spaces
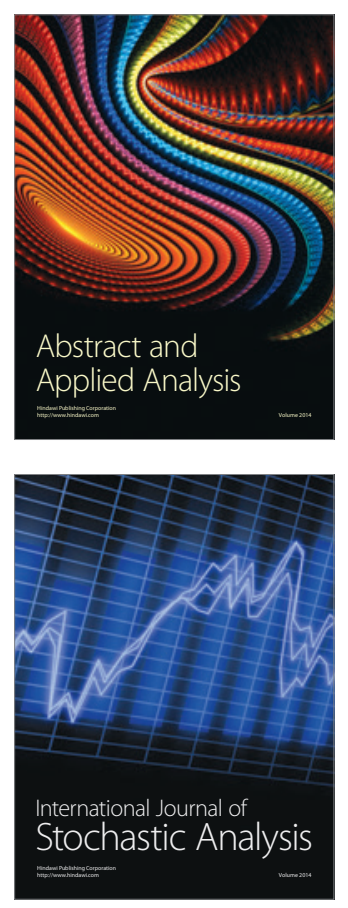

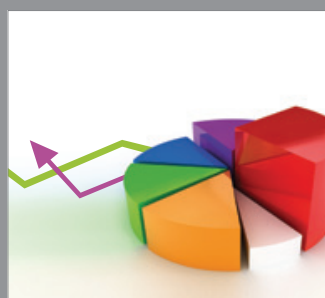

ournal of

Probability and Statistics

Promensencen
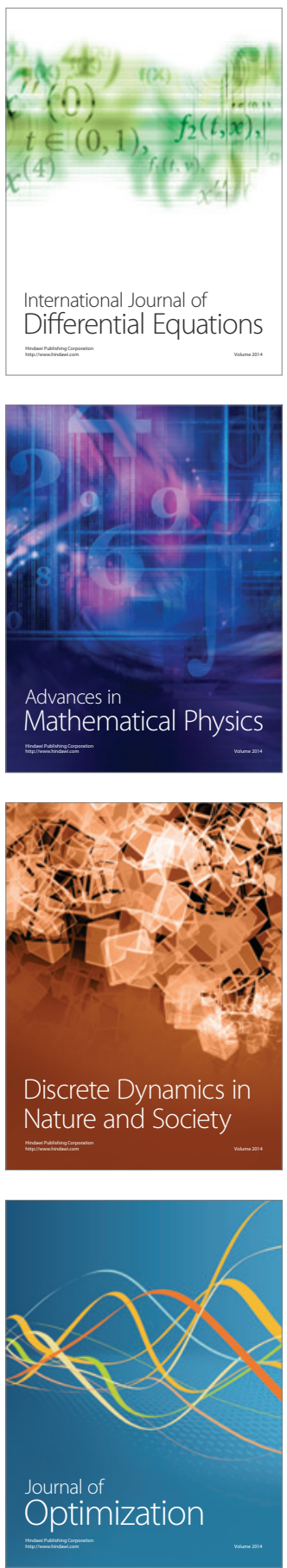